\title{
A Novel Short-Time Fourier Transform-Based Fall Detection Algorithm Using 3-Axis Accelerations
}

\author{
Isu Shin, Jongsang Son, Soonjae Ahn, Jeseong Ryu, Sunwoo Park, Jongman Kim, \\ Baekdong Cha, Eunkyoung Choi, and Youngho Kim
}

Department of Biomedical Engineering, Yonsei University, Wonju 220-710, Republic of Korea

Correspondence should be addressed to Youngho Kim; younghokim@yonsei.ac.kr

Received 27 February 2015; Accepted 27 March 2015

Academic Editor: Xin-She Yang

Copyright (c) 2015 Isu Shin et al. This is an open access article distributed under the Creative Commons Attribution License, which permits unrestricted use, distribution, and reproduction in any medium, provided the original work is properly cited.

\begin{abstract}
The short-time Fourier transform- (STFT-) based algorithm was suggested to distinguish falls from various activities of daily living (ADLs). Forty male subjects volunteered in the experiments including three types of falls and four types of ADLs. An inertia sensor unit attached to the middle of two anterior superior iliac spines was used to measure the 3-axis accelerations at $100 \mathrm{~Hz}$. The measured accelerations were transformed to signal vector magnitude values to be analyzed using STFT. The powers of low frequency components were extracted, and the fall detection was defined as whether the normalized power was less than the threshold (50\% of the normal power). Most power was observed at the frequency band lower than $5 \mathrm{~Hz}$ in all activities, but the dramatic changes in the power were found only in falls. The specificity of $1-3 \mathrm{~Hz}$ frequency components was the best (100\%), but the sensitivity was much smaller compared with $4 \mathrm{~Hz}$ component. The $4 \mathrm{~Hz}$ component showed the best fall detection with $96.9 \%$ sensitivity and $97.1 \%$ specificity. We believe that the suggested algorithm based on STFT would be useful in the fall detection and the classification from ADLs as well.
\end{abstract}

\section{Introduction}

Falls are often caused by various factors such as an impaired balance function or cardiovascular diseases, which result in the deterioration of quality of life [1]. The occurrence of falls increases in magnitude, as the number of elderly people increases in many nations throughout the world. According to World Health Organization, 1.6-3.0 of 10,000 individuals are hospitalized due to falls in the population over 60 years of age in Australia, Canada, and the United Kingdom of Great Britain and Northern Ireland [2]; thus, fall-related injuries are considered as one of major public health priorities. In this regard, many researchers have exerted to develop an exercise protocol [3] or a fall-related injury prevention system [4]. In particular, since positive exercise effects could be expected after quiet long period such as 12 weeks and since the exercise does not always give an escape from a fall, the automatic (preimpact) detection of a fall is considered as a promising solution to prevent the fall-related injury.
In order to effectively prevent fall-related injuries, it is highly desirable to detect a fall prior to impact. To accomplish this, many researchers have been interested in specific changes in accelerations or angular velocities of a human segment under a fall condition [5-7]. Wu measured the horizontal and vertical velocities of the trunk segment using a $3 \mathrm{D}$ motion capture system and found out that (1) the magnitude of both velocities increased dramatically during the falling phase, reaching up to 2-3 times compared to normal velocities, and (2) the increase of both velocity magnitudes usually occurred simultaneously [5]. She suggested that changes in the magnitude and the timing of the magnitude of both velocities could be used to distinguish fall movements from normal activities of daily living (ADLs) during the descending phase of the fall. However, such videobased approach might be limited in terms of cost and most importantly portability. Recently, a wearable sensor has been widely used to measure inertia properties due to its great portability. Bourke et al. proposed a fall detection algorithm 
by thresholding of the vertical velocity of the trunk from a wearable inertial sensor and provided a possibility of stand-alone fall detection systems without an optical motion capture system, showing $100 \%$ accurate distinguishing of falls from ADLs [6]. However, a threshold-based algorithm generally has a high sensitivity but relatively low specificity. This might cause more serious problems than a fall itself; thus, a more robust algorithm is required than a threshold-based one.

A few groups have attempted human activity classification using frequency analysis. Barralon et al. [8] determined the most proper frequency-based analysis method to detect walking activities using the root-mean square of the threeaxis acceleration signals worn on the chest and suggested the short-time Fourier transform (STFT) due to the shortest calculation time compared with the discrete wavelet transform (DWT) or the continuous wavelet transform. Godfrey et al. [9] utilized the DWT into investigating if accurate mobility monitoring and activity classification, including posture transition, can be achieved using a single chestmounted sensor. As a result, the range of both sensitivity and specificity was $86-92 \%$ for young healthy subjects in a controlled setting and $83-89 \%$ for elderly healthy subjects in a home environment. To the best of our knowledge, although using an inertia sensor unit for the fall detection has been recently studied, evaluating the performance of frequencybased algorithms has been less explored before.

In this study, the STFT-based fall detection algorithm was suggested using the three-axis acceleration signals measured at the middle of the two anterior superior iliac spines under various ADLs and simulated falling condition. Then, both sensitivity and specificity were calculated in order to evaluate the performance of the proposed algorithm.

\section{Methods}

2.1. Participants and Experimental Design. Forty healthy male volunteers (age: $23.4 \pm 4.4$ years; height: $172.0 \pm 7.1 \mathrm{~cm}$; weight: $68.7 \pm 8.9 \mathrm{~kg}$ ) with no musculoskeletal diseases participated in this study. Before participating in the study, the subjects were informed about the purpose of the study and the experimental protocol and provided the written informed consents which were approved by the Yonsei University Research Ethics Committee (1041849-201308-BM-001-01). An inertia sensor, MPU-9150 (Invensens, USA), was used to measure the 3 -axis acceleration ( $x$ : mediolateral; $y$ : superoinferior; and $z$ : anteroposterior) at $100 \mathrm{~Hz}$. The inertia sensor was attached to the middle of the two anterior superior iliac spines. Each subject fell by simply relaxing to the side, back, or front on a soft foam mattress for five times, and four different ADLs (gait, sit-to-stand, stand-to-sit, and sit-to-lying) were conducted for five times.

2.2. Short-Time Fourier Frequency-Based Fall Detection Algorithm. Signal vector magnitude (SVM) was calculated from the measured 3-axis acceleration as follows:

$$
s[n]=\sqrt{a_{x}[n]^{2}+a_{y}[n]^{2}+a_{z}[n]^{2}} \quad n=0, \ldots, N-1,
$$

where $s$ is the discrete SVM signal, $a$ the measured acceleration, $n$ the time frame, and $N$ the total length of the signal $s$. The subscript $x, y$, or $z$ indicates the direction. Then, the STFT was conducted using the calculated SVM data $(s[n])$ as follows:

$$
\begin{array}{r}
S[m, \omega]=\sum_{n=0}^{N-1} s[n] w[m-n] \cdot e^{-j \omega m} \\
\\
\omega=0, \ldots, 50 \mathrm{~Hz},
\end{array}
$$

where $S[m, \omega]$ is the STFT output of $s[n]$ to be analyzed, $m$ the time parameter, and $\omega$ the frequency parameter. In this case, $m$ is discrete and $\omega$ is continuous, but both variables become discrete and quantized because in most typical applications the STFT is performed on a computer using the fast Fourier transform (FFT) as follows:

$$
S[\omega]=\sum_{n=0}^{N-1} s[n] \cdot e^{-j \omega}
$$

where $S[\omega]$ is the FFT output of $s[n]$ to be transformed. $w$ in (2) is the Hamming function with the window length $(L)$ of 30 (corresponding to $300 \mathrm{~ms}$ due to the sampling rate of $100 \mathrm{~Hz}$ ) as follows:

$$
w[n]=\alpha-\beta \cos \left(\frac{2 \pi n}{L-1}\right)
$$

where $\alpha$ and $\beta$ are the constants. The constants are approximations of values $\alpha=0.54$ (i.e., 25/46) and $\beta=0.46$ (i.e., 21/46), which cancel the first side-lobe of the Hanning window by placing a zero at frequency $5 \pi /(L-1)$ [10]. The overlapping length was fixed $L-1$ (i.e., 29). Because the SVM signal $s$ is real, the effect of FFT is to evenly distribute the total power on both halves of the input. This means that the FFT output will be symmetric; thus, there are effectively two bins for each frequency, and the magnitude of each bin is effectively half of the actual expected value. The STFT output was corrected as follows:

$$
S[m, \omega]=\sqrt{|2 * S[m, \omega]|} .
$$

Changes in power $S[m, \omega]$ of low frequency components such as $1,2,3,4$, and $5 \mathrm{~Hz}$ were extracted and then were normalized by each of mean values $M_{\omega}$ within the first $100 \mathrm{~ms}$ because the power corresponding to each frequency was different according to movements as follows:

$$
\begin{aligned}
S_{N}[m, \omega] & =\frac{S[m, \omega]}{M_{\omega}} \omega=1,2,3,4,5 \mathrm{~Hz}, \\
M_{\omega} & =\frac{\sum_{m=0}^{99} S[m, \omega]}{100},
\end{aligned}
$$

where $S_{N}[m, \omega]$ is the normalized power. The fall would be expected if the normalized power at each of such frequencies 


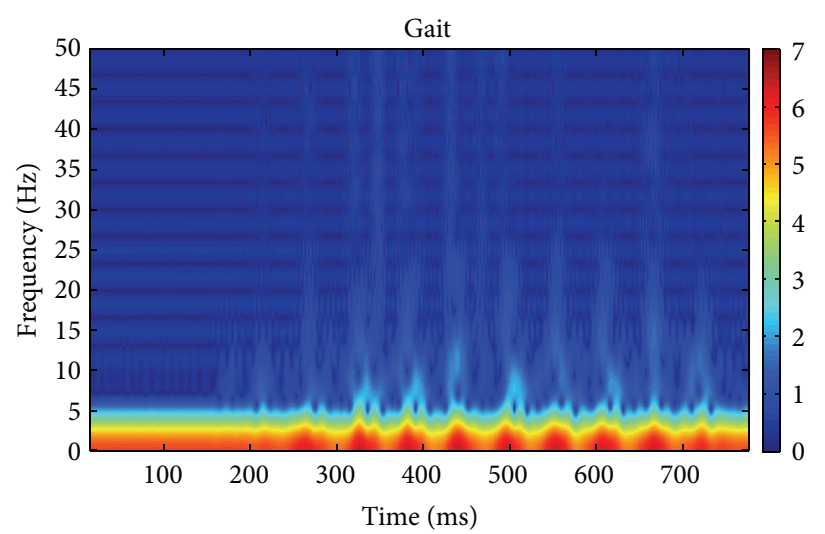

(a)

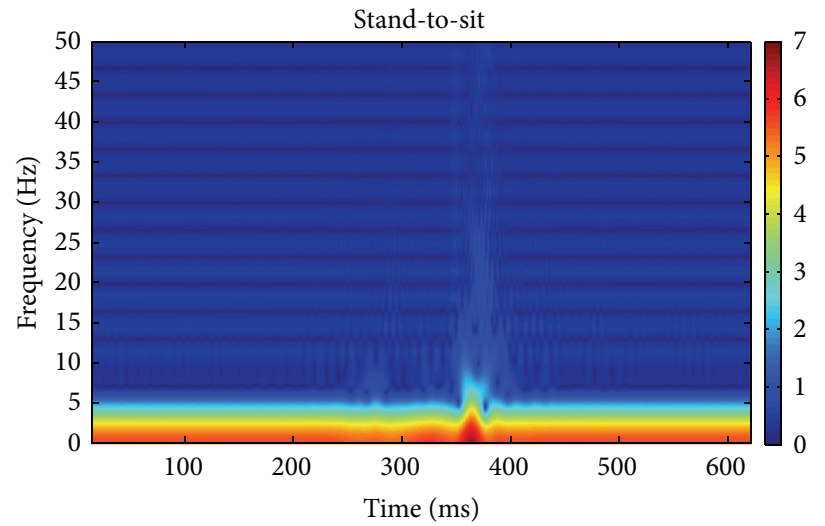

(c)

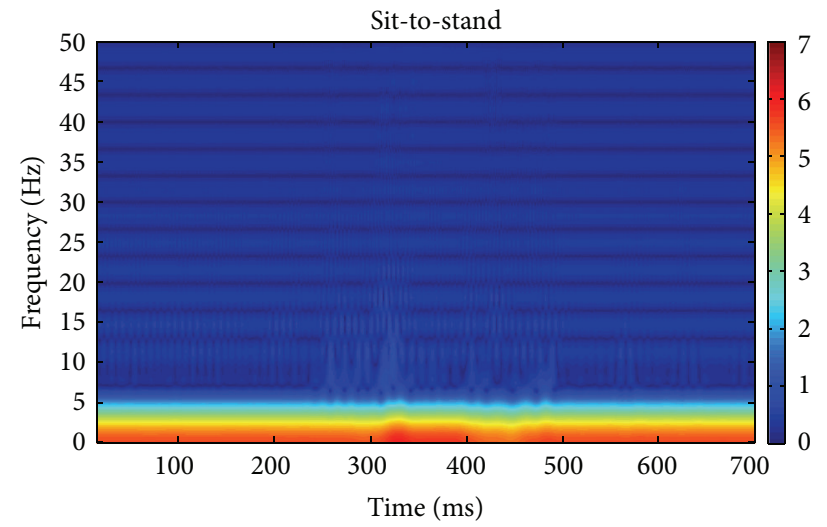

(b)

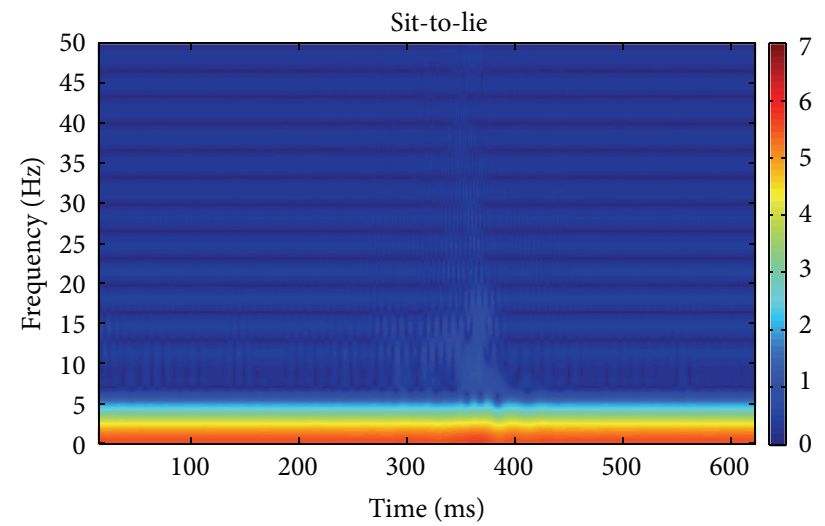

(d)

FIGURE 1: Representative STFT plots for ADLs: gait (a); sit-to-stand (b); stand-to-sit (c); and sit-to-lie (d).

was less than 0.5 of the normalized power (i.e., $S_{N}[m, \omega]<$ 0.5 ). To estimate the performance of fall detection using the STFT, sensitivity and specificity were calculated as follows:

$$
\begin{aligned}
\text { Sensitivity }(\%)= & \frac{\text { True positives }}{\text { True positives + False negatives }} \\
& \times 100 \%, \\
\text { Specificity }(\%)= & \frac{\text { True negatives }}{\text { True negatives + False positives }} \\
& \times 100 \%,
\end{aligned}
$$

where true positives are the number of cases when the algorithm correctly decided a fall as a fall, false negatives the number of cases when the algorithm correctly decided a fall as an ADL, true negatives the number of cases when the algorithm correctly decided an ADL as an ADL, and false positives the number of cases when the algorithm correctly decided an ADL as a fall. All data analysis was performed using MATLAB R2012b (The MathWorks Inc., USA).

\section{Results and Discussion}

Most power was shown at lower frequency components, especially lower than $5 \mathrm{~Hz}$ (over $82 \%$ on average) as shown
TABLE 1: Comparison of sensitivity and specificity according to different frequency components.

\begin{tabular}{lccccc}
\hline & \multicolumn{5}{c}{ Frequency components } \\
& $1 \mathrm{~Hz}$ & $2 \mathrm{~Hz}$ & $3 \mathrm{~Hz}$ & $4 \mathrm{~Hz}$ & $5 \mathrm{~Hz}$ \\
\hline Sensitivity (\%) & 54.1 & 61.5 & 79.7 & 96.9 & 65.8 \\
Specificity (\%) & 100 & 100 & 100 & 97.1 & 83.9 \\
\hline
\end{tabular}

in Figure 1 (for four different ADLs) and Figure 2 (for three types of falls). The power was dramatically changed during falls, but not during ADLs in the frequency band. The normalized power signals during ADLs and falls are shown in Figures 3 and 4, respectively. The peak power at each frequency component was delayed around $2 \mathrm{~ms}$ after the fall impact due to the impact absorption by the soft foam mattress. However, it seems negligible because the purpose of this study was to detect a fall prior to impact. Interestingly, the normalized power values were less than the threshold during falls but not during ADLs. This difference showed a possibility that the developed STFT-based algorithm would be applied for fall detection.

Table 1 shows the performance of the developed algorithm. The specificity of $1-3 \mathrm{~Hz}$ frequency components was 


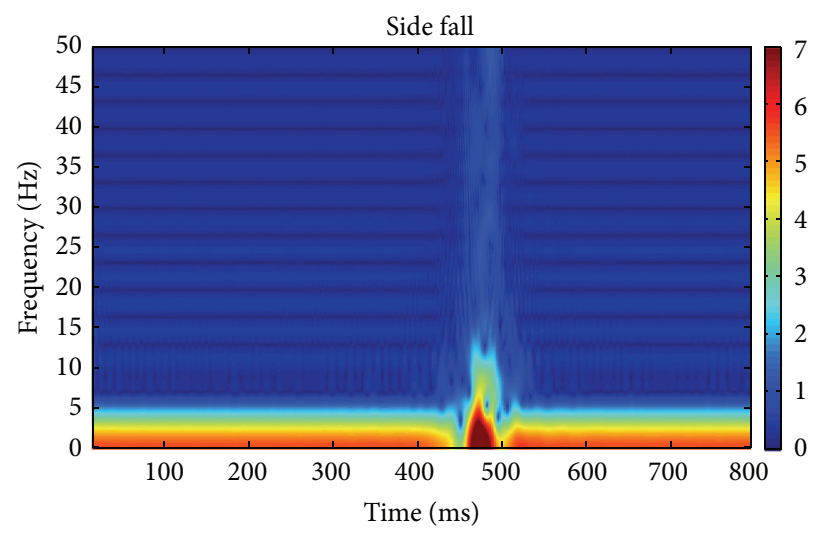

(a)

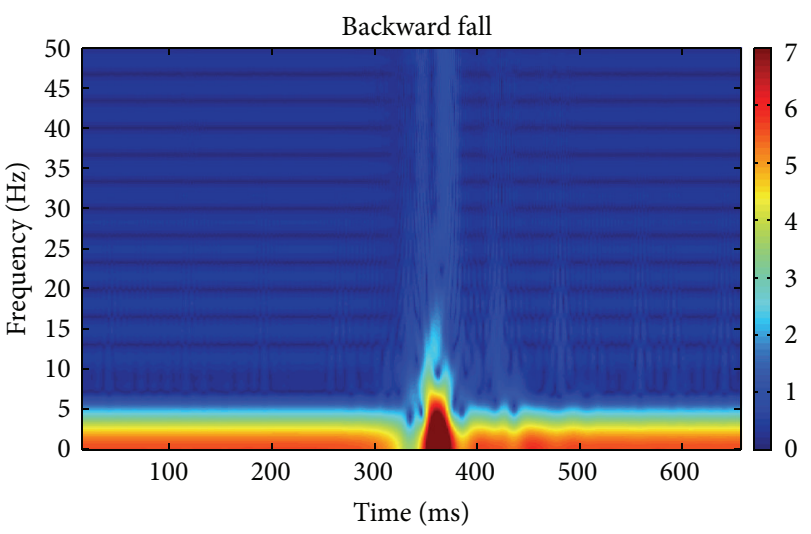

(b)

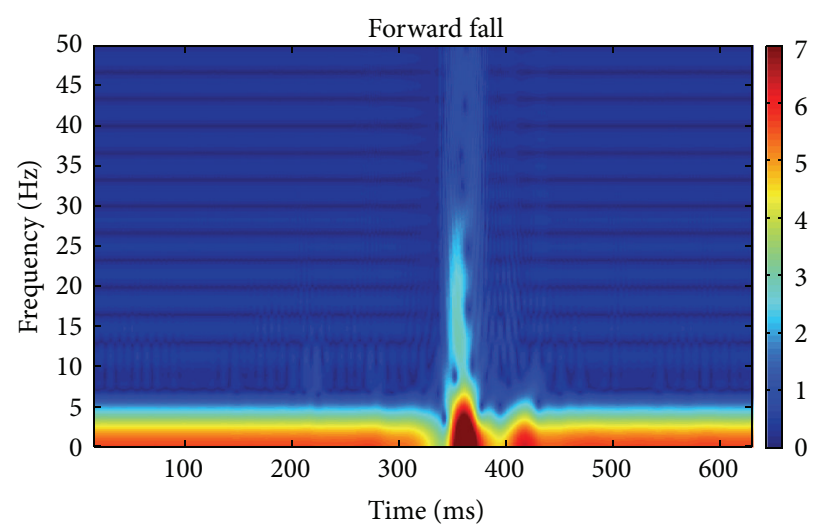

(c)

FIGURE 2: Representative STFT plots for falls: side fall (a); backward fall (b); and forward fall (c).

the best $(100 \%)$, but the sensitivity was low $(<80 \%)$. The results at $5 \mathrm{~Hz}$ showed the low performance with the sensitivity of $65.8 \%$ and the specificity of $83.9 \%$. Although the specificity of $4 \mathrm{~Hz}$ component was rather smaller than $100 \%$ (97.1\%), it showed the best sensitivity (96.9\%) compared with the other frequency components. These imply that $4 \mathrm{~Hz}$ frequency component would be appropriate for the fall detection with the relatively good sensitivity and specificity. This is also supported by the previous study where the results of fast Fourier transform of acceleration signals obtained during a fall showed a considerable power at the frequency band ranged at 3-4 Hz [11].

Many researchers have suggested a fall detection algorithm using angular velocities or accelerations. However, their algorithm decided lying or stand-to-sit as a fall incorrectly (i.e., low specificity) [5, 7]. However, the developed algorithm showed generally high specificity compared with the previous studies. This might be acceptable because it is clearly possible to generate similar acceleration amplitudes in nonfall events, even though the dramatic change in amplitudes of accelerations can be considered as characteristics of a fall event. In this regard, Yavuz et al. [11] investigated accelerations in frequency domain to distinguish falls from normal actions and suggested that the wavelet-based fall detection method showed better performance than the existing threshold method. Moreover, they proposed that focusing on the frequency components of the acceleration could lead to better performance of distinguishing falls from normal activities than the amplitude. The similar results were found in this study, showing that the normalized power at $4 \mathrm{~Hz}$ would be promising to distinguish falls from ADLs.

This study might be limited due to healthy male subjects participating. However, this is not an avoidable problem in the current environment, because a potential risk is highly expected when the elderly people perform the simulated falls even on a soft foam mattress. Instead of the direct measurement from the simulated experiments, the problem could be solved by monitoring the long-term activities of fallers.

This study included the small number of ADLs. However, the ADLs in this study have been considered as typical activities in the field of the evaluation of fall detection algorithms $[5,7]$. Interestingly, the developed algorithm showed better performance of the fall detection with the relatively good 


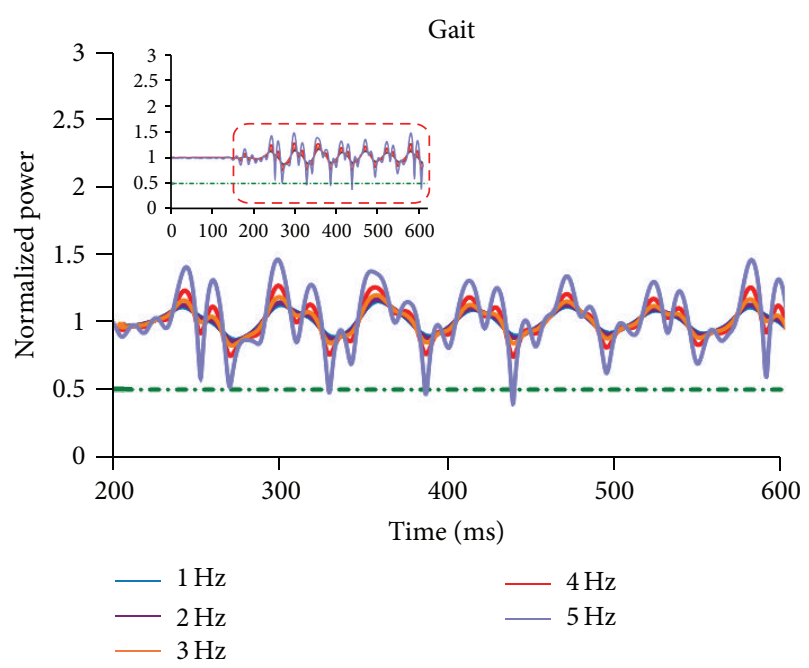

(a)

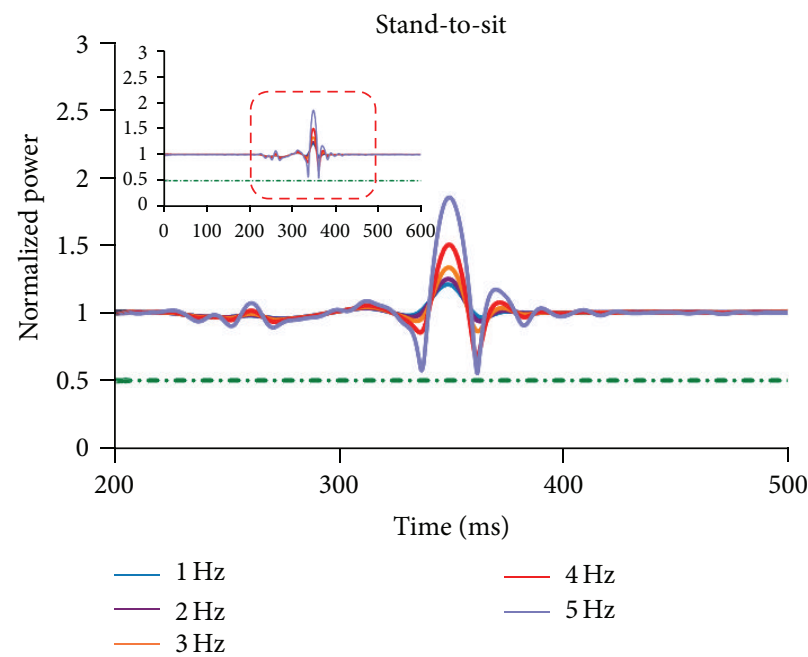

(c)

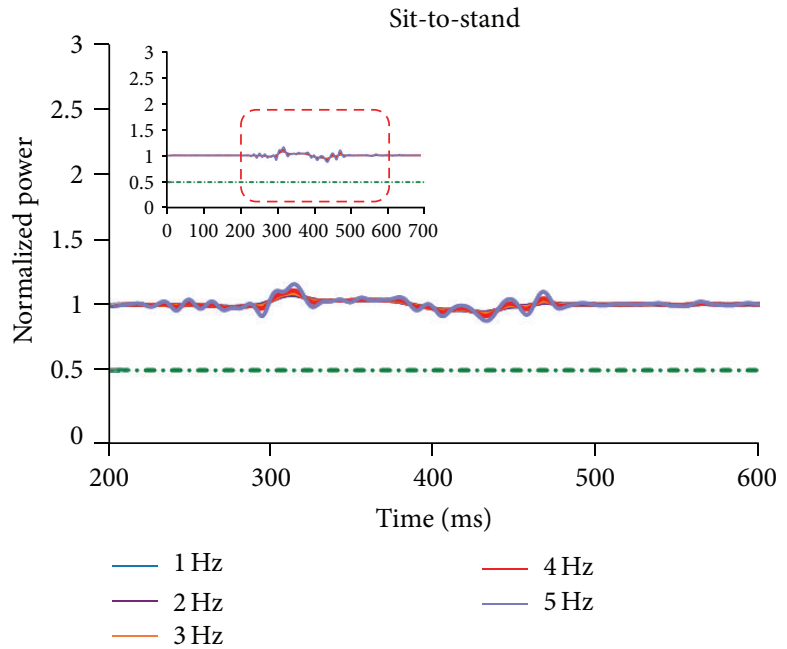

(b)

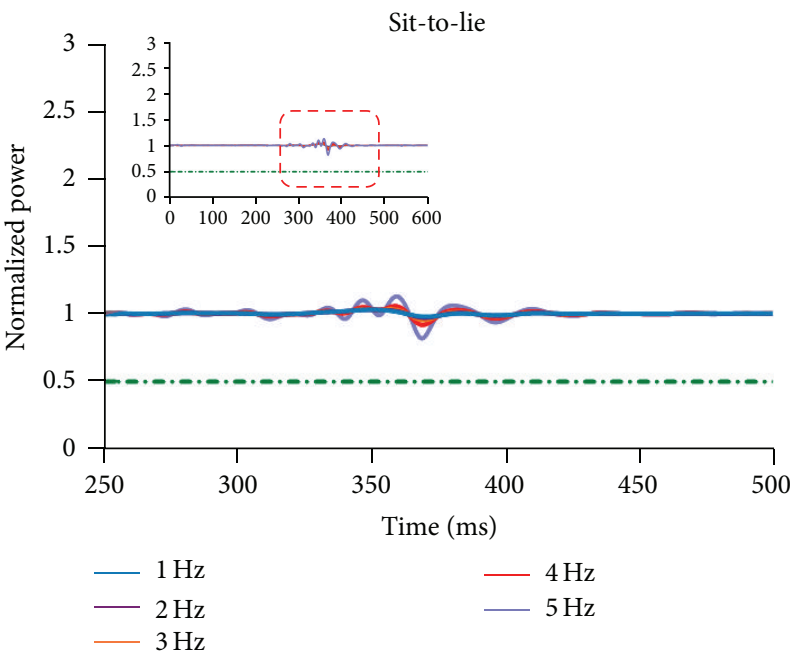

(d)

FIgURE 3: Representative normalized power plots for ADLs: gait (a); sit-to-stand (b); stand-to-sit (c); and sit-to-lie (d). The vertical dashed line indicates the fall impact and the horizontal dashed-dot-line the threshold (i.e., 0.5).

sensitivity and specificity than that of the previous studies. Nevertheless, further studies would be required to confirm whether the developed algorithm would be useful to the other activities.

There are various parameters in performing the STFT, but most parameters such as the window function, the window length, and the overlapping length were fixed. This might be a negligible problem, because the normalized power at $4 \mathrm{~Hz}$ showed better outcomes than the other conditions in spite of the same STFT operation. Banos et al. [12] identified that the window length between 0.25 and $3.25 \mathrm{~s}$ might be optimal for the recognition of whole body movements. Moreover, they suggested that shorter window sizes would be better to recognize fall or epileptic seizure detections. These results imply the importance of the window length for the signal segmentation. Thus, it would be also required to find out the relationship between the performance and changes in the factors in further studies.

In conclusion, this study aimed to develop the STFTbased fall detection algorithm. As a result, the best fall detection could be accomplished with $4 \mathrm{~Hz}$ frequency component, showing $96.9 \%$ sensitivity and $97.1 \%$ specificity. Though combining various sensors (i.e., gyro sensor, accelerometer, or tilt sensor) can be a potential alternative to detect falls with high sensitivity and specificity, it might require a multistep process and complex algorithm. However, the developed algorithm showed the relatively high sensitivity and specificity with only 3 -axis accelerations. Therefore, we believe that the suggested 


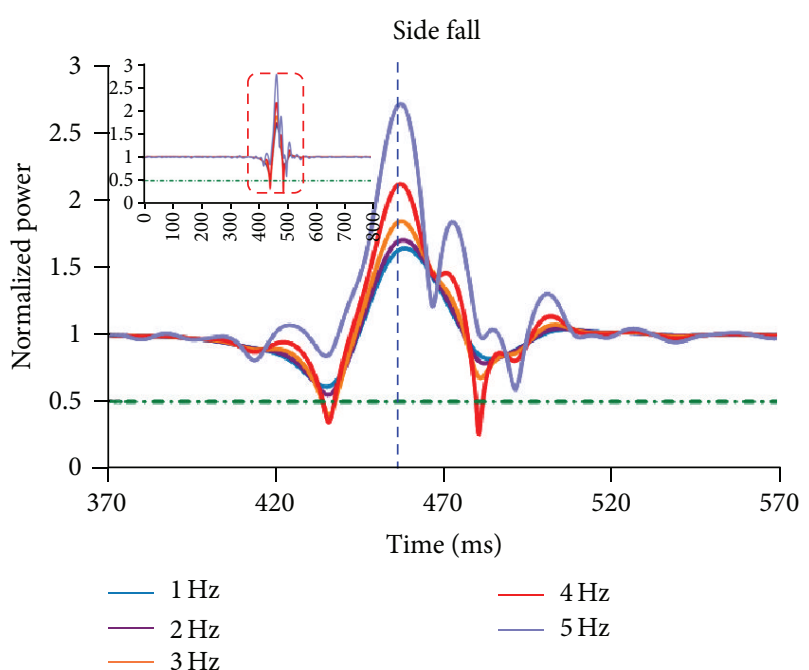

(a)

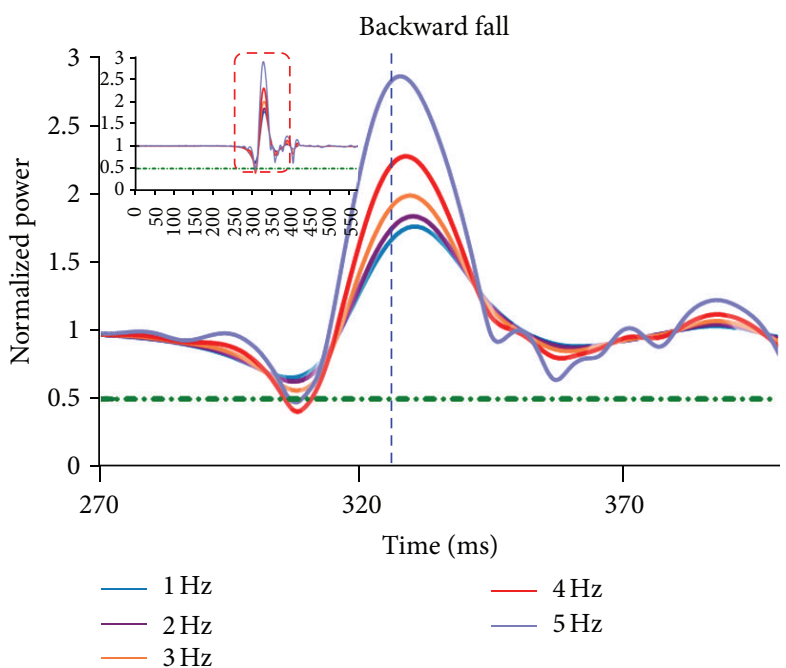

(b)

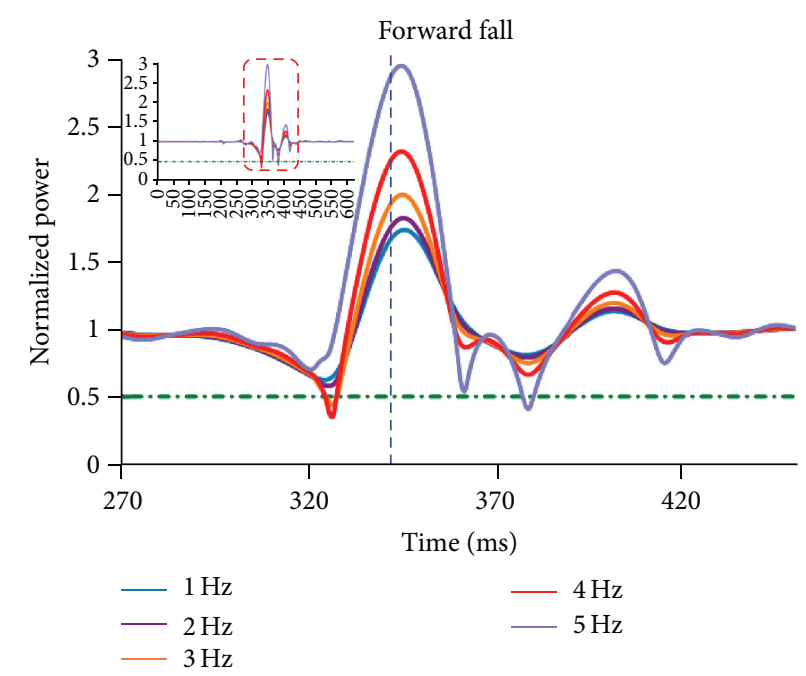

(c)

FIGURE 4: Representative normalized power plots for falls: side fall (a); backward fall (b); and forward fall (c). The vertical dashed line indicates the fall impact, and the horizontal dashed-dot-line the threshold (i.e., 0.5).

algorithm would be useful for the fall detection and the classification from ADLs as well even when there are only 3axis accelerations.

\section{Conflict of Interests}

The authors declare that there is no conflict of interests regarding the publication of this paper.

\section{Authors' Contribution}

J. Son and I. Shin contributed to study concept and design. I. Shin, J. Kim, B. Cha, S. Ahn, and S. Park contributed to acquisition of data. I. Shin, J. Son, J. Ryu, E. Choi, and Y. Kim contributed to analysis and interpretation of data. I. Shin, J. Son, and Y. Kim contributed to writing of the paper.

\section{Acknowledgments}

This research was financially supported by the Ministry of Education (MOE) and National Research Foundation of Korea (NRF) through the Human Resource Training Project for Regional Innovation (no. 2013H1B8A2032194) and was also supported by the Sports Promotion Fund of Seoul Olympic Sports Promotion Foundation from Ministry of Culture, Sports and Tourism (s07201212022013).

\section{References}

[1] M. Suzuki, N. Ohyama, K. Yamada, and M. Kanamori, "The relationship between fear of falling, activities of daily living and quality of life among elderly individuals," Nursing \& Health Sciences, vol. 4, no. 4, pp. 155-161, 2002. 
[2] World Health Organization, WHO Global Report on Falls Prevention in Older Age, chapter 1, World Health Organization, Lyon, France, 2007.

[3] L. Z. Rubenstein, K. R. Josephson, P. R. Trueblood et al., "Effects of a group exercise program on strength, mobility, and falls among fall-prone elderly men," Journals of Gerontology-Series A Biological Sciences and Medical Sciences, vol. 55, no. 6, pp. M317-M321, 2000.

[4] P. Kannus, H. Sievänen, M. Palvanen, T. Järvinen, and J. Parkkari, "Prevention of falls and consequent injuries in elderly people," The Lancet, vol. 366, no. 9500, pp. 1885-1893, 2005.

[5] G. Wu, "Distinguishing fall activities from normal activities by velocity characteristics," Journal of Biomechanics, vol. 33, no. 11, pp. 1497-1500, 2000.

[6] A. K. Bourke, K. J. O’Donovan, and G. ÓLaighin, “The identification of vertical velocity profiles using an inertial sensor to investigate pre-impact detection of falls," Medical Engineering \& Physics, vol. 30, no. 7, pp. 937-946, 2008.

[7] M. N. Nyan, F. E. H. Tay, A. W. Y. Tan, and K. H. W. Seah, "Distinguishing fall activities from normal activities by angular rate characteristics and high-speed camera characterization," Medical Engineering \& Physics, vol. 28, no. 8, pp. 842-849, 2006.

[8] P. Barralon, N. Vuillerme, and N. Noury, "Walk detection with a kinematic sensor: frequency and wavelet comparison," in Proceedings of the 28th Annual International Conference of the IEEE Engineering in Medicine and Biology Society (EMBS '06), pp. 1711-1714, New York, NY, USA, September 2006.

[9] A. Godfrey, R. Conway, D. Meagher, and G. ÓLaighin, "Direct measurement of human movement by accelerometry," Medical Engineering \& Physics, vol. 30, no. 10, pp. 1364-1386, 2008.

[10] F. J. Harris, "On the use of windows for harmonic analysis with the discrete Fourier transform," Proceedings of the IEEE, vol. 66, no. 1, pp. 51-83, 1978.

[11] G. Yavuz, M. Kocak, G. Ergun et al., "A smart phone based fall detector with online location support," in Proceedings of the International Workshop on Sensing for App Phones, pp. 31-35, Zurich, Switzerland, 2010.

[12] O. Banos, J.-M. Galvez, M. Damas, H. Pomares, and I. Rojas, "Window size impact in human activity recognition," Sensors, vol. 14, no. 4, pp. 6474-6499, 2014. 


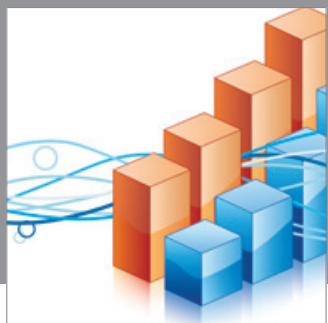

Advances in

Operations Research

mansans

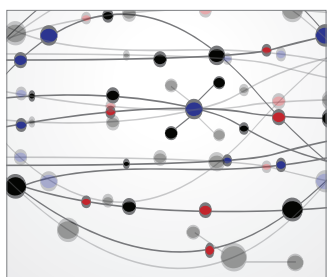

The Scientific World Journal
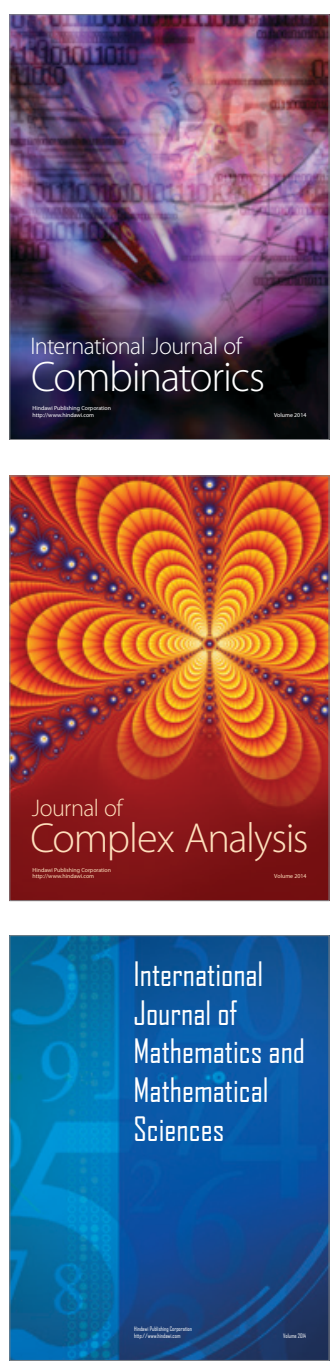
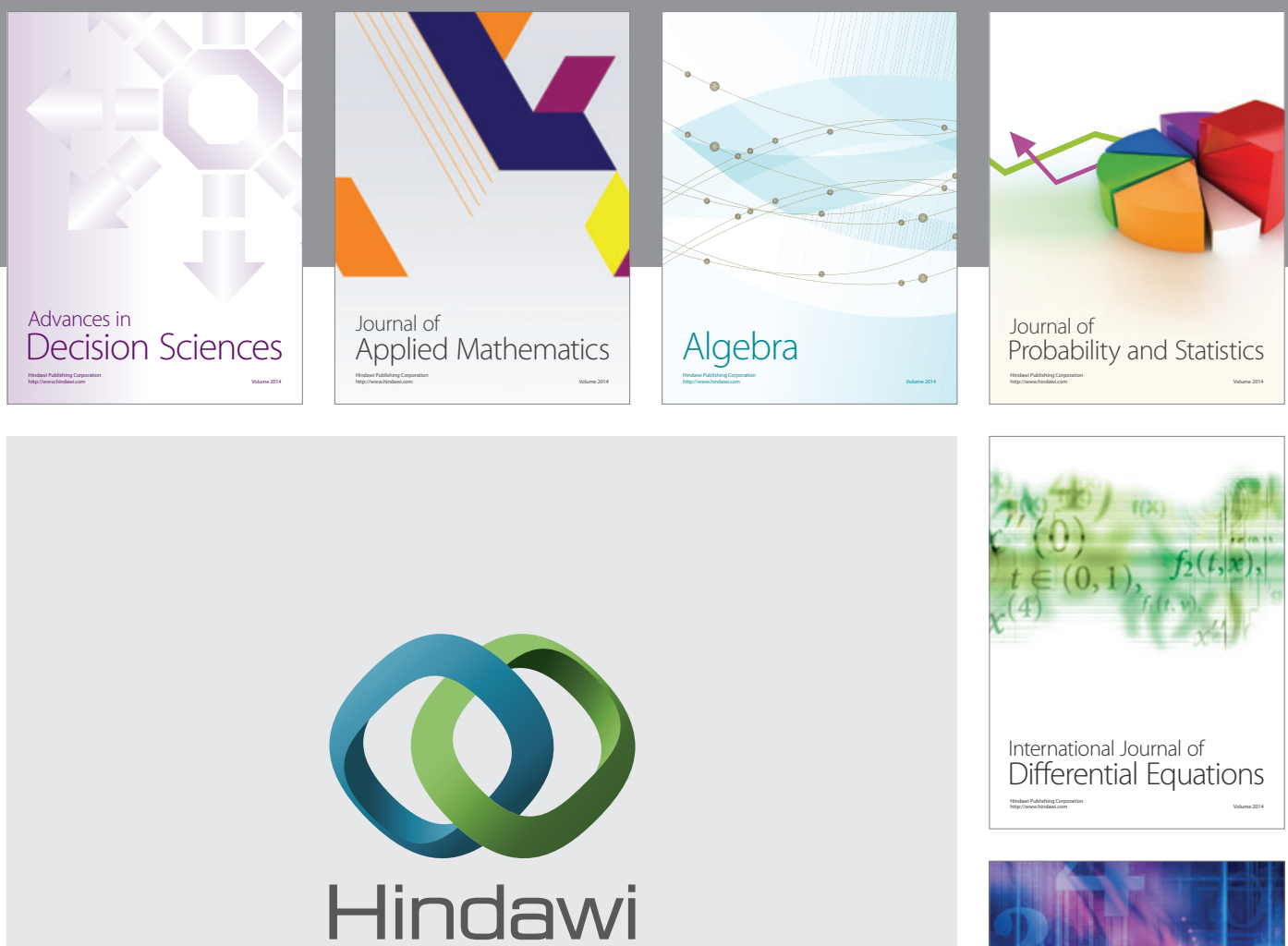

Submit your manuscripts at http://www.hindawi.com
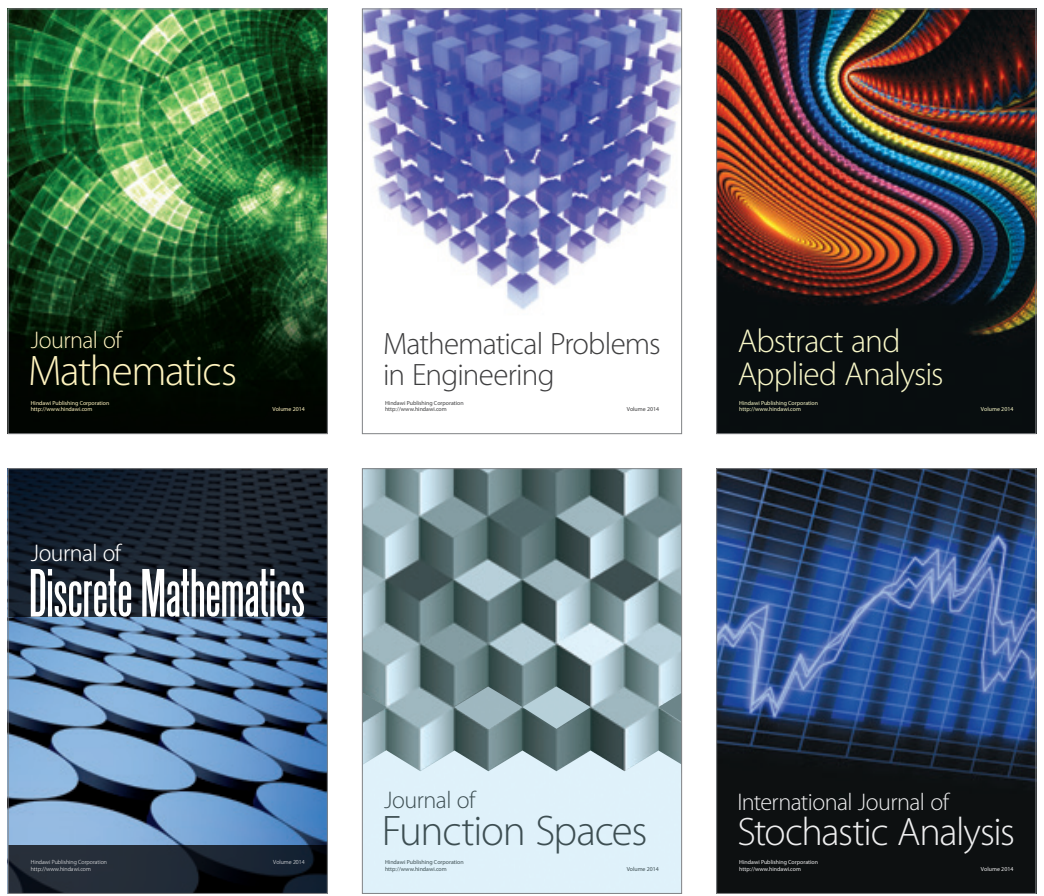

Journal of

Function Spaces

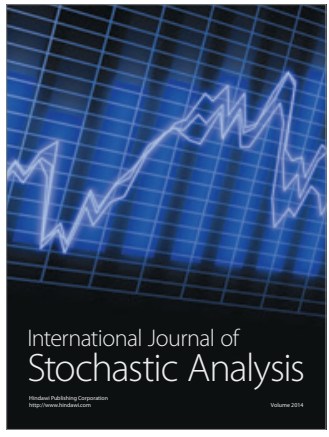

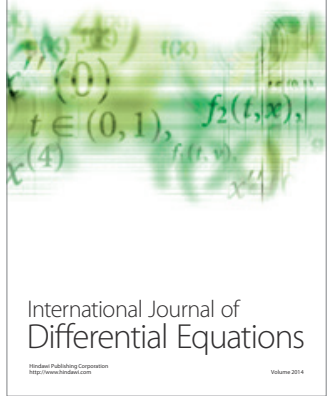
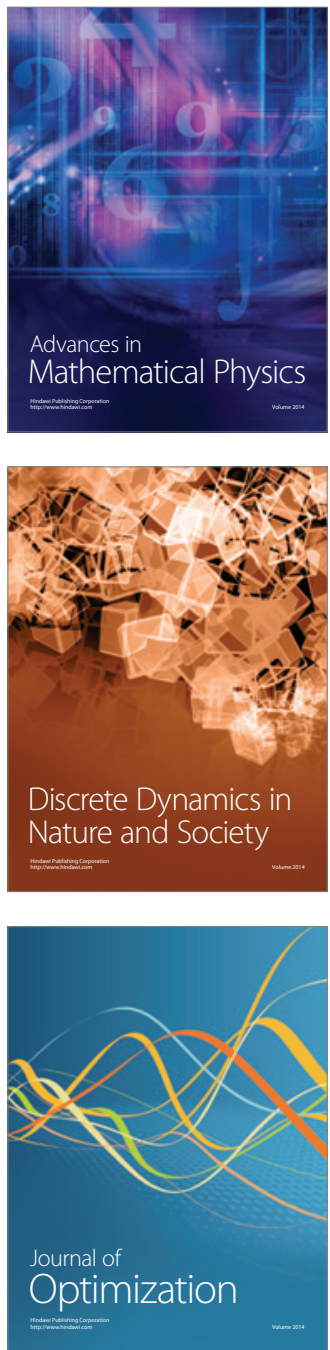